\title{
Optimal Selection and Effort in a Fishery on a Stock with Cannibalistic Behaviour - The Case of the Northeast Arctic Cod Fisheries
}

\author{
Arne Eide* and Arild Wikan** \\ * Norwegian College of Fishery Science, University of Troms $\emptyset$, Norway (arne.eide@nfh.uit.no) \\ ** Harstad University College, Norway
}

\begin{abstract}
We explore a fishery targeting the mature part of a stock, while bycatching the immature part. Both targeted catch and bycatch may have positive market values. Cost of effort is supposed to be a function of the selection properties of the fishing gears. Mature fish is assumed to cannibalise on the immature part of the stock. A bioeconomic model of the problem is set up and it is shown that the model possesses a unique nontrivial stable equilibrium and moreover that both cannibalism and catch act as stabilising factors. Necessary conditions for a bioeconomic optimum are also provided. The model is applied on the Northeast Arctic cod stock where time series of stock biomasses, catches and fishing mortality rates covering the period 1946-2000 have been used to estimate the model parameter values. The economic parameters have been estimated on the basis of previous open access to the fishery, assuming bioeconomic equilibrium. It is shown that bioeconomic optimum could not be obtained at any discount rate, without reducing the rate of fishing mortality, first of all on the mature part of the cod population.
\end{abstract}

Keywords: Bioeconomics, Cannibalism, Cod fishery, Selective fishery

\section{INTRODUCTION}

As documented by Myers and co-workers (Myers et al. 1990) [1] several commercially important fish stocks show highly year to year fluctuations. The Northeast Arctic cod stock is one of these stocks and there is a lot of evidence of such behaviour also in the past, also before the development of industrial cod fisheries (see for example Hjort 1914 [2] and Øiestad 1994 [3]). Long time observed catch fluctuations and cod stock estimates from 1946 to 2000 indicate a close connection between biomass fluctuation and age composition within the stock (Anon., 2001) [4].

In most periods strong year-classes seem to dominate the stock, while a more harmonised year-class composition occurs in other periods. Shifts in yearclass composition seem to influence the stock fluctuation in biomass and the variations is not necessarily reflecting variations in individual growth parameters. Another possible explanation is that the cod stock avoids or minimises such changes in individual growth parameters through cannibalistic behaviour and indeed, the cannibalistic behaviour of cod has been verified through stomach analysis (Bogstad et al. 1994) [5].
This has important dynamical consequences. We have earlier shown that in the interplay between increasing fecundity and increasing cannibalism pressure, the former turns out to be a destabilising effect whiles the latter tends to stabilize [6]. Predation on the stock by other species may also stabilise the dynamics, but there are important cases where predation and cannibalism act differently [7]. Other examples of dynamical consequences of cannibalism may be found in Gurtin and Levin (1982) [8], Cushing (1991) [9], van der Bosch and Gabriel (1997) [10] and Magnússon (1999) [11]. Cushing describes the stabilising self-regulation property of cannibalism [9] and Kohlmeier and Ebenhöh elaborates the stabilising role further also in a predator-prey context [12]. The latter includes cannibalism in the net growth expressed by one differential equation covering a cannibalistic predator.

There is also a vast literature on bioeconomic preypredator models, see for example Hannesson (1983) [13], Ragozin and Brown (1985) [14], Wilen and Brown (1986) [15], Flaaten (1991) [16], Brock and Xepapadeas (2004) [17].

Unlike the papers quoted above, the scope of this paper is to identify social optimal equilibriums for the Northeast Arctic cod fishery by taking the cannibalistic behaviour of the stock into consideration. The biological model used is a symbiotic model and consists 
of two differential equations describing a cannibalistic relationship between mature and immature cod. Furthermore, a linear harvest equation and simple revenue and cost equations are applied. Biological and economic parameters are estimated from a time series covering the period 1946-2000.

Fleet dynamics are not included in the model presented, as the selective properties of fishing gears and fishing effort produced are considered being control variables and management means.

\section{THE MODEL}

Let $x(t)$ and $y(t)$ be the mature and the immature parts of a population at time $t$ respectively, and assume further that the dynamics of $x$ and $y$ may be described by the nonlinear system

$$
\dot{x}=g_{1}(x, y) \quad \dot{y}=g_{2}(x, y)
$$

where

$$
\begin{aligned}
& g_{1}(x, y)=r x\left(1-\frac{x}{a y}\right) \\
& g_{2}(x, y)=s y\left(1-\frac{y}{b x}\right)-k x y
\end{aligned}
$$

(1) may be regarded as a minor modification of the prey-predator model presented by May and coworkers (May et al. 1979) [18], including a positive symbiotic relationship in the prey-predator interaction. The constants $a, b, r, s$ and $k$ are all assumed to be positive. $r$ and $s$ are the intrinsic growth rates of the mature and immature part of the population respectively. The relation $\dot{x}=g_{1}(x, y)$ expresses that the natural equilibrium level of the mature stock is directly related to the abundance of immature by the proportionality factor $a$. Similarly $\dot{y}=g_{2}(x, y)$ expresses that the natural equilibrium level of the immature stock relates to the mature stock by the proportionally factor $b$. The relationships expressed by $a$ and $b$ cover the non-cannibalistic net growth interaction, including the recruitment of surviving immature to the mature fraction of the stock, and the recruitment of immature as a function of the spawning biomass. Cushing [9] points out the double effect cannibalism has on the adult (mature); both by affecting immature survival to become mature and providing the mature with an additional source of energy. $\dot{y}=g_{2}(x, y)$ contains a somewhat crude form of cannibalism relation, assuming the immature part of the population to be consumed at a rate proportional to their density per mature biomass. $k$ will be referred to as the cannibalism parameter. The overall effect on the immature may be more easily seen when $g_{2}(x, y)$ is reformulated to $(s-k x) y\left(1-\frac{s y}{b x(s-k x)}\right), \quad$ where the expression $(s-k x)$ corresponds to the intrinsic growth rate of a logistic growth equation and $b x(s-k x) / s$ the equilibrium biomass. Both are functions of the mature stock, the intrinsic growth rate being reduced by cannibalism (represented by the parameter $k$ ), while two mechanisms affect the equilibrium biomass, the recruitment (represented by the parameter $b$ ) and cannibalism.

The model was initially proposed by Eide [19] and has later been applied in several bioeconomic studies where cannibalism plays an important role [20] [21] [22].

Let us now extend model (1) by including catch. Assume that $\mathrm{x}$ is the target of harvest with a bycatch of $\mathrm{y}$, depending on the selection properties of the fishing gears. The selection parameter is $\alpha, \alpha \geq 0$. The special case gives no bycatch of $y$, while represents the situation of no selection and consequently equal fishing mortalities $f$ for $x$ and $y$. Hence, the annual harvest of the targeted mature part of the stock, $h_{1}$, and the bycatch, $h_{2}$, are described by

$$
\begin{aligned}
& h_{1}=h_{1}(f, x)=f x \\
& h_{2}=h_{2}(\alpha, f, y)=\alpha f y
\end{aligned}
$$

Including harvest the model is expressed by

$$
\begin{aligned}
& \dot{x}=g_{1}(x, y)-f x \\
& \dot{y}=g_{2}(x, y)-\alpha f y
\end{aligned}
$$

The unique nontrivial equilibrium of (4) is found to be

$$
(\bar{x}, \bar{y})=
$$

$$
\left(\begin{array}{l}
\frac{1}{k}\left[s-\alpha f-\frac{r s}{a b(r-f)}\right], \\
\frac{r}{a k(r-f)}\left[s-\alpha f-\frac{r s}{a b(r-f)}\right]
\end{array}\right)
$$

where $s>\alpha f, r>f$ and $s-\alpha f>r s[a b(r-f)]^{-1}$ are necessary constraints in order to ensure biological acceptable equilibrium (non-negative biomasses). Denoting the Jacobian of (4), evaluated at equilibrium by $J$, the trace $(t r)$ and determinant (det) of $J$ are

$$
\begin{aligned}
& \operatorname{tr}(J)=-(r-f)-\frac{r s}{a b(r-f)}<0 \\
& \operatorname{det}(J)=(r-f)\left(s-\alpha f-\frac{r s}{a b(r-f)}\right)>0
\end{aligned}
$$


Hence, the equilibrium $(\bar{x}, \bar{y})$ is locally stable. From a dynamic point of view this finding allow us to conclude that increased harvest may not give birth to nonstationary behaviour, a result which to a great extent is in accordance with the findings of Wikan and Eide [6].

Table 1.The Northeast Arctic cod stock biomass and catches in million tons 1946-2000. The figures are from ICES' Advisory Committee on Fisheries Management (Anon. 2001) [4] tables 3.7 (catches), 3.19 (stock biomass) and 3.23 (maturity).

\begin{tabular}{|c|c|c|c|c|c|c|}
\hline \multirow[b]{2}{*}{ Year $(t)$} & \multicolumn{2}{|c|}{ Stock size (biomass in mill. tones) } & \multicolumn{2}{|c|}{ Catch (mill. tones) } & \multicolumn{2}{|c|}{ Fishing mortality rate } \\
\hline & Mature $(x)$ & Immature $(y)$ & Mature $(h x)$ & Immature (hy) & Mature $(f)$ & Immature $(\alpha f)$ \\
\hline 1946 & 1.1206 & 3.3866 & 0.2633 & 0.4222 & 0.2350 & 0.1247 \\
\hline 1947 & 1.1740 & 2.7283 & 0.4688 & 0.4959 & 0.3993 & 0.1818 \\
\hline 1948 & 1.0264 & 2.8562 & 0.3510 & 0.5176 & 0.3420 & 0.1812 \\
\hline 1949 & 0.7360 & 2.5528 & 0.2563 & 0.5503 & 0.3482 & 0.2156 \\
\hline 1950 & 0.6206 & 2.5292 & 0.2444 & 0.4284 & 0.3938 & 0.1694 \\
\hline 1951 & 0.5741 & 3.0474 & 0.1940 & 0.5263 & 0.3379 & 0.1727 \\
\hline 1952 & 0.5271 & 3.4114 & 0.2428 & 0.6951 & 0.4606 & 0.2038 \\
\hline 1953 & 0.4010 & 3.8533 & 0.1425 & 0.5209 & 0.3554 & 0.1352 \\
\hline 1954 & 0.4350 & 3.9139 & 0.1691 & 0.7196 & 0.3887 & 0.1839 \\
\hline 1955 & 0.3512 & 3.2905 & 0.1526 & 0.9268 & 0.4345 & 0.2817 \\
\hline 1956 & 0.3034 & 3.1074 & 0.1421 & 1.1425 & 0.4684 & 0.3677 \\
\hline 1957 & 0.2103 & 2.6487 & 0.0900 & 0.7023 & 0.4280 & 0.2651 \\
\hline 1958 & 0.1978 & 2.1979 & 0.0830 & 0.6019 & 0.4196 & 0.2739 \\
\hline 1959 & 0.4382 & 2.2912 & 0.1806 & 0.6197 & 0.4121 & 0.2705 \\
\hline 1960 & 0.3890 & 2.0169 & 0.1629 & 0.4343 & 0.4188 & 0.2153 \\
\hline 1961 & 0.4104 & 2.1368 & 0.2026 & 0.5113 & 0.4937 & 0.2393 \\
\hline 1962 & 0.3167 & 1.9697 & 0.1574 & 0.5785 & 0.4970 & 0.2937 \\
\hline 1963 & 0.2118 & 1.7560 & 0.1257 & 0.6335 & 0.5935 & 0.3608 \\
\hline 1964 & 0.1892 & 1.3421 & 0.0989 & 0.3270 & 0.5227 & 0.2436 \\
\hline 1965 & 0.1036 & 1.6769 & 0.0458 & 0.2991 & 0.4421 & 0.1784 \\
\hline 1966 & 0.1224 & 2.7595 & 0.0499 & 0.3425 & 0.4077 & 0.1241 \\
\hline 1967 & 0.1318 & 3.2925 & 0.0628 & 0.4620 & 0.4765 & 0.1403 \\
\hline 1968 & 0.2306 & 3.2669 & 0.0914 & 0.9045 & 0.3964 & 0.2769 \\
\hline 1969 & 0.1546 & 2.7351 & 0.0886 & 1.0495 & 0.5731 & 0.3837 \\
\hline 1970 & 0.2281 & 1.9394 & 0.1175 & 0.7180 & 0.5151 & 0.3702 \\
\hline 1971 & 0.3167 & 1.4845 & 0.1546 & 0.4008 & 0.4882 & 0.2700 \\
\hline 1972 & 0.3523 & 1.7130 & 0.1895 & 0.2886 & 0.5379 & 0.1685 \\
\hline 1973 & 0.3380 & 2.8488 & 0.1733 & 0.4363 & 0.5127 & 0.1532 \\
\hline 1974 & 0.1669 & 2.3150 & 0.0839 & 0.7231 & 0.5027 & 0.3124 \\
\hline 1975 & 0.1440 & 2.1793 & 0.0678 & 0.6522 & 0.4708 & 0.2993 \\
\hline 1976 & 0.1738 & 2.0396 & 0.0755 & 0.6082 & 0.4344 & 0.2982 \\
\hline 1977 & 0.3473 & 1.7767 & 0.1740 & 0.6734 & 0.5010 & 0.3790 \\
\hline 1978 & 0.2460 & 1.6184 & 0.1469 & 0.4947 & 0.5972 & 0.3057 \\
\hline 1979 & 0.1778 & 1.0343 & 0.0980 & 0.2649 & 0.5512 & 0.2561 \\
\hline 1980 & 0.1102 & 0.8233 & 0.0590 & 0.2400 & 0.5354 & 0.2915 \\
\hline 1981 & 0.1699 & 0.8910 & 0.0942 & 0.2437 & 0.5544 & 0.2735 \\
\hline 1982 & 0.3315 & 0.4938 & 0.1622 & 0.1283 & 0.4893 & 0.2598 \\
\hline 1983 & 0.3328 & 0.4865 & 0.1916 & 0.1323 & 0.5757 & 0.2719 \\
\hline 1984 & 0.2556 & 0.7402 & 0.1444 & 0.1484 & 0.5649 & 0.2005 \\
\hline 1985 & 0.1966 & 0.9909 & 0.0977 & 0.2047 & 0.4969 & 0.2066 \\
\hline 1986 & 0.1731 & 1.5661 & 0.0914 & 0.3319 & 0.5280 & 0.2119 \\
\hline 1987 & 0.1203 & 1.1331 & 0.0724 & 0.4392 & 0.6018 & 0.3876 \\
\hline 1988 & 0.2053 & 0.8044 & 0.1178 & 0.3171 & 0.5738 & 0.3942 \\
\hline 1989 & 0.1970 & 0.7770 & 0.0904 & 0.2461 & 0.4589 & 0.3167 \\
\hline 1990 & 0.3446 & 0.7320 & 0.0985 & 0.1112 & 0.2858 & 0.1519 \\
\hline 1991 & 0.6847 & 1.0702 & 0.1927 & 0.1425 & 0.2814 & 0.1332 \\
\hline 1992 & 0.8847 & 1.3533 & 0.2960 & 0.2037 & 0.3346 & 0.1505 \\
\hline 1993 & 0.7461 & 2.0052 & 0.2769 & 0.2974 & 0.3711 & 0.1483 \\
\hline 1994 & 0.6137 & 1.9007 & 0.3248 & 0.4394 & 0.5292 & 0.2312 \\
\hline 1995 & 0.5093 & 1.6134 & 0.2640 & 0.4738 & 0.5184 & 0.2937 \\
\hline 1996 & 0.5804 & 1.3109 & 0.2856 & 0.4360 & 0.4921 & 0.3326 \\
\hline 1997 & 0.5763 & 1.2744 & 0.3248 & 0.4373 & 0.5636 & 0.3431 \\
\hline 1998 & 0.3961 & 1.2146 & 0.2326 & 0.3558 & 0.5872 & 0.2929 \\
\hline 1999 & 0.2645 & 1.0585 & 0.1477 & 0.3388 & 0.5584 & 0.3201 \\
\hline 2000 & 0.2264 & 1.0854 & 0.1285 & 0.2836 & 0.5676 & 0.2613 \\
\hline
\end{tabular}

In the next section we assume $x$ and $y$ to represent the mature and the immature fractions of the Northeast Arctic cod stock respectively. Catch data and stock biomasses from the period 1946-2000 are employed in order to estimate the model parameters $r, s, a, b$ and $k$ in (1), (2).

\section{BIOLOGICAL GROWTH PARAMETER ESTIMATES}

As the data set presented in Table 1 is a discrete time representation of the dynamic system, (1), (2) has to be rewritten as discrete time equations in 
order to estimate parameter values of the five a priori positive parameters $(a, b, r, s$, and $k)$. Total annual growth of the mature and immature fraction (corresponding to differential equations (2)) may then be expressed as

$$
\Delta x(t)=x(t+1)-x(t)
$$

and

$$
\Delta y(t)=y(t+1)-y(t) .
$$

Mature and immature biomasses of year $t+1$ (including an error term $u$ ) are obtained from the data set of year $t$, assuming the discrete version of model (6). The model's biomass estimates at given parameter values are denoted $\hat{x}$ and $\hat{y}$.

$$
\begin{aligned}
& \hat{x}(t+1)= \\
& (1+r) x(t)-\frac{r \cdot x(t)^{2}}{a \cdot y(t)}-f(t) \cdot x(t)+u_{x}(t)
\end{aligned}
$$

and

$$
\begin{aligned}
& \hat{y}(t+1)= \\
& (1+s) y(t)-\frac{s \cdot y(t)^{2}}{b \cdot x(t)}-k \cdot x(t) \cdot y(t)- \\
& \alpha(t) \cdot f(t) \cdot y(t)+u_{y}(t),
\end{aligned}
$$

for $t \in[1946,2000]$.

It should however be noted that the biomasses in Table 1 in this study are treated as data even though they really are estimates calculated on the basis of catch data, and an accounting method known as Virtual Population Analysis (VPA, or XSA which is the extended version also including additional features to utilise other information through tuning methodology [4]. In this study the XSA estimation method is regarded as a measuring method rather than model estimates, as the stock biomasses are measured through methodology build on relative consistency within year classes. Uncertainties regarding actual biomass level are real, but not relevant for our model, as biomass indexes, not necessarily real values, are sufficient to parameterise the model.

As in [6] system parameters are estimated simultaneously by identifying parameter sets of local minimums of the squared sum of total (mature and immature) biomass

$$
S S S Q=\sum_{t=1946}^{2000}\{(\hat{x}(t)+\hat{y}(t))-(x(t)+y(t))\}^{2},
$$

while only accepting non-negative parameter values. The best fit parameter set is presented in Table 2 and illustrated in Fig. (1). [19] employs a weighted sum of squared residuals in the mature and immature biomasses instead of the flat sum in (11). Separating into mature and immature fractions may however become a critical factor in the estimation process and since this separation may be uncertain (in particular for the first part of the time series [4]), a method focusing total biomass rather that the biomass fractions of immature and mature biomasses, was preferred in this study.

Table 2. Estimated parameters of equations (2) found by minimising (11), applying data of Table 1.

\begin{tabular}{llr}
\multicolumn{1}{c}{ Parameter/SSSQ } & \multicolumn{1}{c}{$\begin{array}{c}\text { Estimated } \\
\text { value }\end{array}$} \\
\hline $\begin{array}{l}\text { Intrinsic growth rate of mature fraction } \\
\text { Proportionality factor of maximum mature } \\
\text { stock }\end{array}$ & $r$ & 0.6867 \\
$\begin{array}{l}\text { Intrinsic growth rate of immature fraction } \\
\text { Proportionality factor of maximum }\end{array}$ & $b$ & 0.3628 \\
$\begin{array}{l}\text { immature stock } \\
\text { Proportionality factor of cannibalism }\end{array}$ & $k$ & 0.5386 \\
\hline Constrained minimum of $S S S Q(11)$ & & 12.3656 \\
\hline Number of years in time series $(n)$ & 0.1653 \\
\hline$S S S Q / n$ & 55 \\
\hline
\end{tabular}

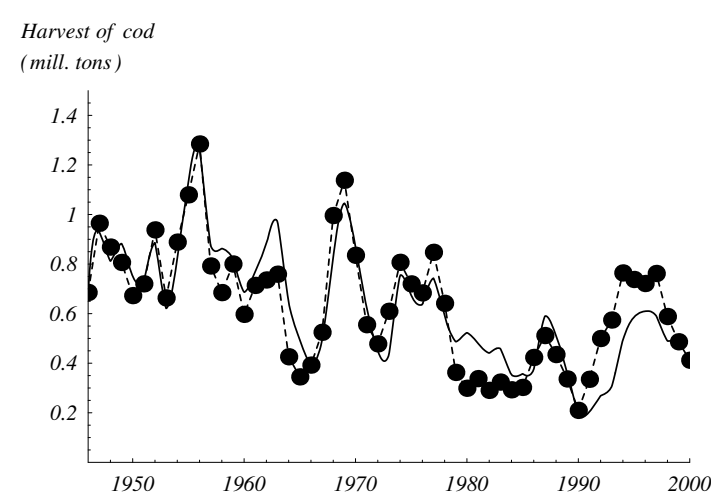

Fig (1). Estimated total catch of mature and immature cod 1946-2000 by use of (1), (2) and (6). Initial stock sizes are taken from 1946 and historical fishing mortalities (Table 1) have been used. The points connected by the dashed curve represent actual annual historical catches (Table 1) over the same period.

The estimation was carried out by numerical methods searching for a regional minimum of (11) within valid parameter space. A program based on internal minimizing function in the software Mathematica ${ }^{\Theta}$ was used in the numerical estimation procedure. The algorithms and Mathematica ${ }^{\odot}$ 
notebook used for parameter estimations may be obtained by contacting the corresponding author.

As shown in Fig. (1) the model has an acceptable fit to historical data, indicating that a large part of the observed fluctuations may be explained by the model. The model phase plot in absence of fishery (Fig. 2) shows natural equilibrium as a stable focus close to the initial stock situation in 1946.

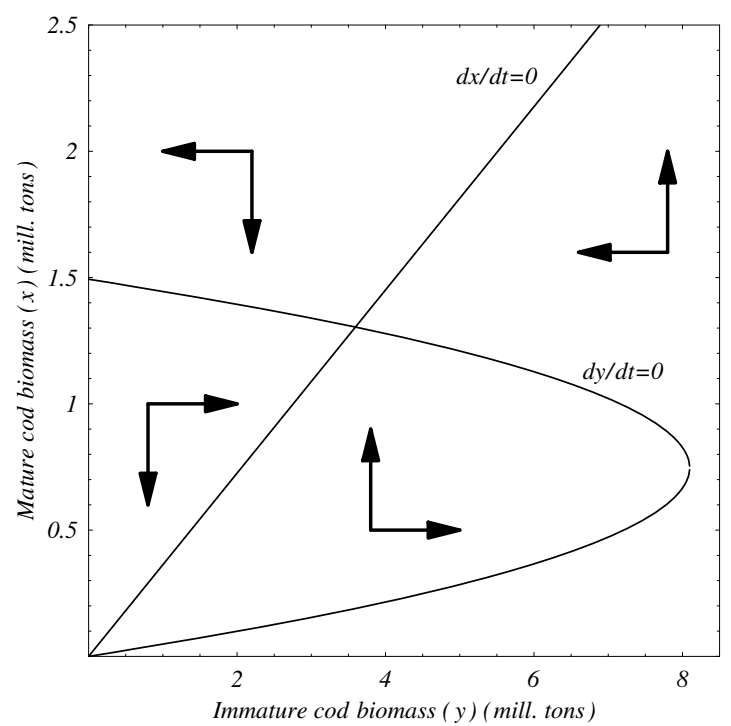

Fig (2). Phase plot of the natural growth system (1) and (2), parameter values from Table 2 . Note that the displayed system does not include fishing mortalities as fleet dynamics is not included.

\section{HARVEST ECONOMY MODEL AND PARAMETER ESTIMATES}

Now turn to the economic aspects of fishing. A simple economic model is set up and parameterised assuming essentially an open access fishery in the past. Available variables for managing the fishery are gear selection and any measures controlling total fishing effort.

Denoting constant unit prices on catch from the two fractions ( $x$ and $y$ ) for $p_{1}$ and $p_{2}$ respectively, total annual revenue $(T R)$ of the fishery may be expressed as

(12) $\operatorname{TR}(\alpha, f, x, y)=p_{1} \cdot h_{1}(f, x)+p_{2} \cdot h_{2}(\alpha, f, y)$,

or equivalently, by use of the definitions of $h_{1}$ and $h_{2}$ (cf. (5)) as

$$
\operatorname{TR}(\alpha, f, x, y)=\left(p_{1} \cdot x+\alpha \cdot p_{2} \cdot y\right) f .
$$

The total cost (TC) simply reflects the cost of producing a certain amount of fishing mortality. Assuming a constant unit cost of fishing effort and a linear relationship between rate of fishing mortality and fishing effort, there will be a constant unit cost of fishing mortality rate. Fishing effort is the fishing activity produced by labour (fisherman) and capital (boat and fishing gear) and the unit cost of effort includes the opportunity costs of these input factors, by which a normal profit is obtained when total revenue covers the total costs. In this case the possibility of a selective fishery is included, so the basic assumption of constant unit cost of effort is related to a given, fixed selection property of the fishing fleet.

Let $c_{1}$ be the unit cost of fishing effort for the selection value $\alpha=1$ (which could be labelled no selection). Assume further that any other positive value of $\alpha$ involves a larger unit cost of fishing effort, as selection is obtained by making the fishing gears less efficient on one or both of the two fractions of the stock, the mature and immature part. Specifically targeting mature or immature fish therefore involves costs both by reducing the probability of fishing the non-targeted part and by increasing the probability of fishing the targeted. The former is obtained by technologically changing the gear (essentially making it less efficient), while the latter is obtained by increasing the inputs in effort production, by which the unit cost of effort is increasing.

Change in unit cost of fishing mortality is given by $c_{2}$. Total cost (including all opportunity costs) will be a function of $f$ and $\alpha$ with a minimum when $\alpha=1$. Therefore, we shall assume throughout the paper the cost function

$$
T C(\alpha, f)=\left(c_{1}+c_{2}(1-\alpha)^{2}\right) f,
$$

which is consistent with the given constraints, where $c_{1}$ and $c_{2}$ are constant cost parameters ( $\left.c_{1} \geq 0, c_{2} \geq 0\right)$. Note that the right hand side of (14) increases as $\alpha$ is increased or decreased from the non-selective value 1

Resource rent obtained from the fishery, $\pi$, is the a function of stock size $(x)$ and effort (here given by the fishing mortality rate $f$ ),

$$
\pi(\alpha, f, x, y)=T R(\alpha, f, x, y)-T C(\alpha, f) .
$$

It is well known that bioeconomic equilibrium of a fishery of one control variable (fishing effort), if existing, is defined by 


$$
\frac{T R}{f}=\frac{\partial T C}{\partial f} .
$$

$T R$ and $T C$ refer to equations (13) and (14). The individual decision maker may be controlled by the selection value $(\alpha)$ and the fishing mortality rate $(f)$ in the fishery. According to equation (14) the unit cost of effort has its minimum when $\alpha=1$, which will be the open access selection value when no capacity constraints and technological regulations are put on the harvesting units.

Equality (16) should therefore be a valid criterion of open access or bioeconomic equilibrium also in this case. Inserting (14) and (15) and substituting $x$ and $y$ by applying the nontrivial equilibrium (7) the bioeconomic equilibrium criterion (16) (when $\alpha=1$ ) is

$$
\begin{aligned}
& p_{2} r(a b(f-r)(f-s)-r s)= \\
& p_{1} r s+a(f-r)\left(a b(f-r)\left(c_{1} k+p_{1}(f-s)\right)\right)
\end{aligned}
$$

(17) is a cubic function of $f$, by which one root will be the bioeconomic equilibrium solution if an open access solution exists.

Table 3. Assumed price parameter values and estimated cost parameter values when $c_{2}=2 \cdot c_{1}$. The cost parameter estimation was done by minimising $\sum_{t=1946}^{2000}[\pi(\alpha(t), f(t), x(t), y(t))]^{2}$.

Parameter

\begin{tabular}{|l|l|r|}
\hline \multicolumn{2}{|c|}{ Parameter } & Value \\
\hline Unit price of mature cod (kilo) & $p_{x}$ & 12.00 \\
\hline Unit price of immature cod (kilo) & $p_{y}$ & 8.00 \\
\hline Minimum unit cost of effort & $c_{1}$ & 7.55 \\
\hline Selection cost & $c_{2}$ & 15.11 \\
\hline
\end{tabular}

In the current model unit cost of effort may not be constant and two unit prices exist on harvest. It is however not obvious how detailed price information over the period (which includes a range of different qualities, changes in market preferences over the period, etc.) should be used in estimating two simple price parameters. Generally mature cod is regarded as a more valuable product than the immature. Furthermore it seems reasonable to assume on average a low or no resource rent over the period. In this analysis prices are assumed to be 12 and 8 NOK per kilo respectively for mature and immature cod, while the cost parameters have been estimated on the basis of assumed prices and the basic assumption of normal profits in the fishery, from the data of Table 1 and profit equation (15).

Since no available information was found on the cost of selection, unit cost of effort in case of $100 \%$

catch selectivity (no immature catch, $\alpha=0$ ) is assumed to be twice the unit cost of no selection, e.g. $c_{2}=2 \cdot c_{1}$. The economic parameter values obtained on the basis of these assumptions are presented in Table 3.

When assuming a constant discount rate $\delta$, present value of the resource rent from the fishery over all time is

$$
P=\int_{t=0}^{\infty} \pi(\alpha(t), f(t), x(t), y(t)) \cdot e^{-\delta \cdot t} \cdot d t
$$

Our next goal is to maximise (18) subject to (4) where $x(t)$ and $y(t)$ are regarded as state variables and $\alpha(t) \in[0, \infty], f(t) \in[0, \infty]$ are the controls. By use of the current value formulation, the Hamiltonian of the problem is

$$
\begin{aligned}
& H^{C}= \\
& \pi(\alpha, f, x, y)+\lambda_{1} \cdot\left(g_{1}(x, y)-f \cdot x\right)+ \\
& \lambda_{2} \cdot\left(g_{2}(x, y)-\alpha \cdot f \cdot x\right)
\end{aligned}
$$

where $\lambda_{i}=\lambda_{i}(t), i=1,2$ are the adjoint functions. Further, from the maximum principle

$$
\begin{aligned}
& \dot{\lambda}_{1}-\delta \cdot \lambda_{1}=-H_{x}{ }^{C} \\
& \dot{\lambda}_{2}-\delta \cdot \lambda_{2}=-H_{y}{ }^{C}
\end{aligned}
$$

(The subscripts $x$ and $y$ denote the derivatives with respect to $x$ and $y$ ). If we additionally suppose optimal controls to be located in the interior of the convex control region, we have

$$
H_{\alpha}{ }^{C}=0 \quad \text { and } \quad H_{f}{ }^{C}=0
$$

Finally, using the fact that the bioeconomic model (4) possesses a unique nontrivial stable equilibrium (5), we find after some algebra from (20) and (21) that the optimal controls $\alpha^{*}$ and $f^{*}$ (at equilibrium) may be obtained from the relations

$$
\frac{x\left(f^{*} y \cdot \pi_{x}+g_{2 x} \cdot \pi_{\alpha}\right)}{y\left(f^{*} \pi_{y}+\alpha^{*} \pi_{\alpha}\right)}-\left(r-f^{*}\right)=\delta
$$

$$
\frac{y\left(f^{*} x \cdot \pi_{y}+\left(f^{*} \pi_{f}-\alpha^{*} \pi_{\alpha}\right) g_{1 y}\right)}{x \cdot \pi_{\alpha}}-\frac{r \cdot s}{a \cdot b\left(r-f^{*}\right)}=\delta
$$

where $x, y, \pi_{x}, \ldots$ must be evaluated at equilibrium. Note that $(22 \mathrm{a}, \mathrm{b})$ are cubic equations in $\alpha^{*}$ and $f^{*}$ and must be solved by means of numerical methods. 


\section{RESULTS}

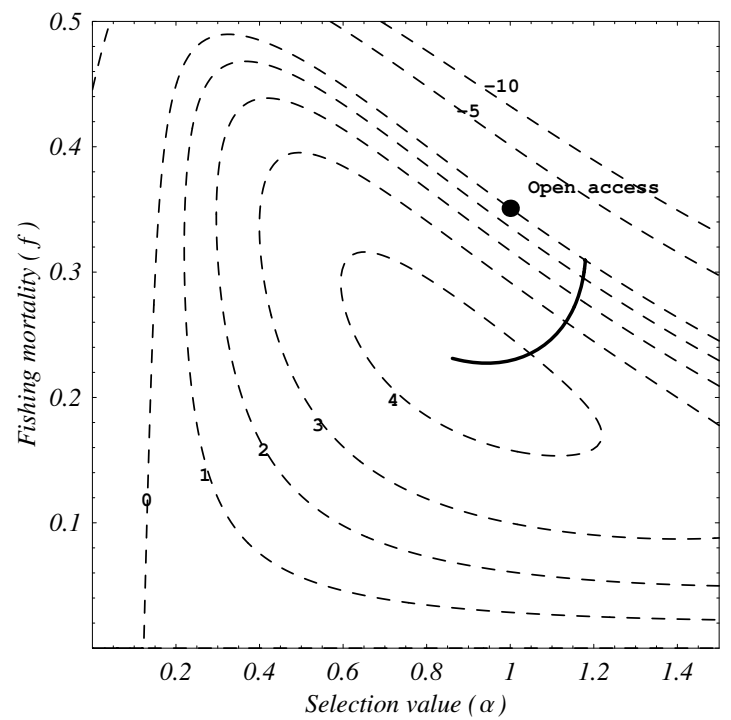

Fig. (3). The dashed curves represent isovalues (of $0,1,2,3$ and 4 billion NOK respectively) of total resource rent of the fishery (equation (15)), while the solid line gives the unique solution of the two optimal equilibrium conditions $(22 \mathrm{a}, \mathrm{b})$ for nonnegative values of the discount rate $(\delta)$, starting at the maximum resource rent location when $\delta=0$, all as functions of the system control variables $\alpha$ and $f$. The point indicates bio-economic equilibrium when $\alpha=1$ (17).

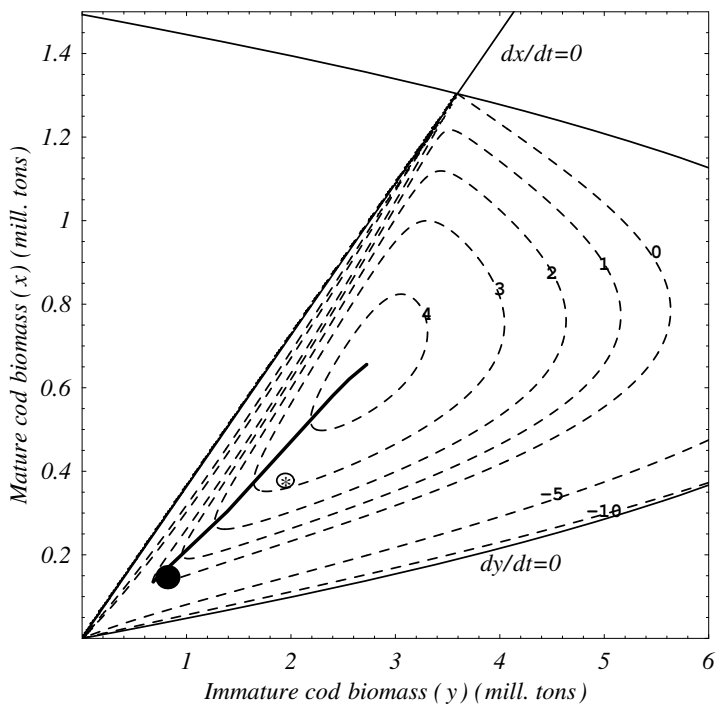

Fig. (4). Phase plot of the system state variables $x$ and $y$ (solid lines, see also Fig. 2) and contour plot of equation (15) at equilibrium (dashed curves) showing isocurves of $-10,-5,0,1,2,3$ and 4 billion NOK, as indicated. As in Fig. (3) the calculated optimal points of different discount rates are represented as a solid line drawn from the maximum point, ending close the open access equilibrium of $\alpha=1$ (filled circle point) when $\delta=\infty$. Average combined biomasses over the period 1946-2000 (from Table 4) are shown by the star surrounded by a circle.

Table 4. Bioeconomic equilibrium (open access solution), equation (17) and optimal conditions (22a, b) for different values of the discount rate $\delta$ as illustrated in Fig. (3). Current state (year 2000) and average values for the period 1946-2000, with resource rent calculation equal annual average, are also provided. $x$ and $y$ in million tons. $\pi$ (annual resource rent from equation (15), while including opportunity cost of labour and capital) in billion NOK.

Calculated Equilibriums

Open access solution
Optimal solution, $\delta=\infty$
Optimal solution, $\delta=0$

Optimal solution, $\delta=0.10$

Optimal solution, $\delta=0.05$

Optimal solution, $\delta=0$

No fishery

\begin{tabular}{ccccc}
$\boldsymbol{\alpha}$ & $\boldsymbol{f}$ & $\boldsymbol{x}$ & $\boldsymbol{y}$ & $\boldsymbol{\pi}$ \\
\hline 1.0000 & 0.3473 & 0.1465 & 0.8169 & 0.0000 \\
\hline 1.1799 & 0.3095 & 0.1354 & 0.6795 & 0.0000 \\
1.1542 & 0.2731 & 0.3041 & 1.3919 & 2.3467 \\
0.9904 & 0.2287 & 0.5807 & 2.3999 & 4.2159 \\
0.9344 & 0.2274 & 0.6204 & 2.5567 & 4.3078 \\
0.8598 & 0.2313 & 0.6557 & 2.7253 & 4.3415 \\
- & 0.0000 & 1.3037 & 3.5932 & 0.0000
\end{tabular}

Historical data

\begin{tabular}{lllllr}
\hline Year 2000 & 0.4604 & 0.5676 & 0.2264 & 1.0854 & -2.9752 \\
\hline Average values (1946-2000) & 0.5316 & 0.4695 & 0.3779 & 1.9462 & 1.4032 \\
\hline
\end{tabular}



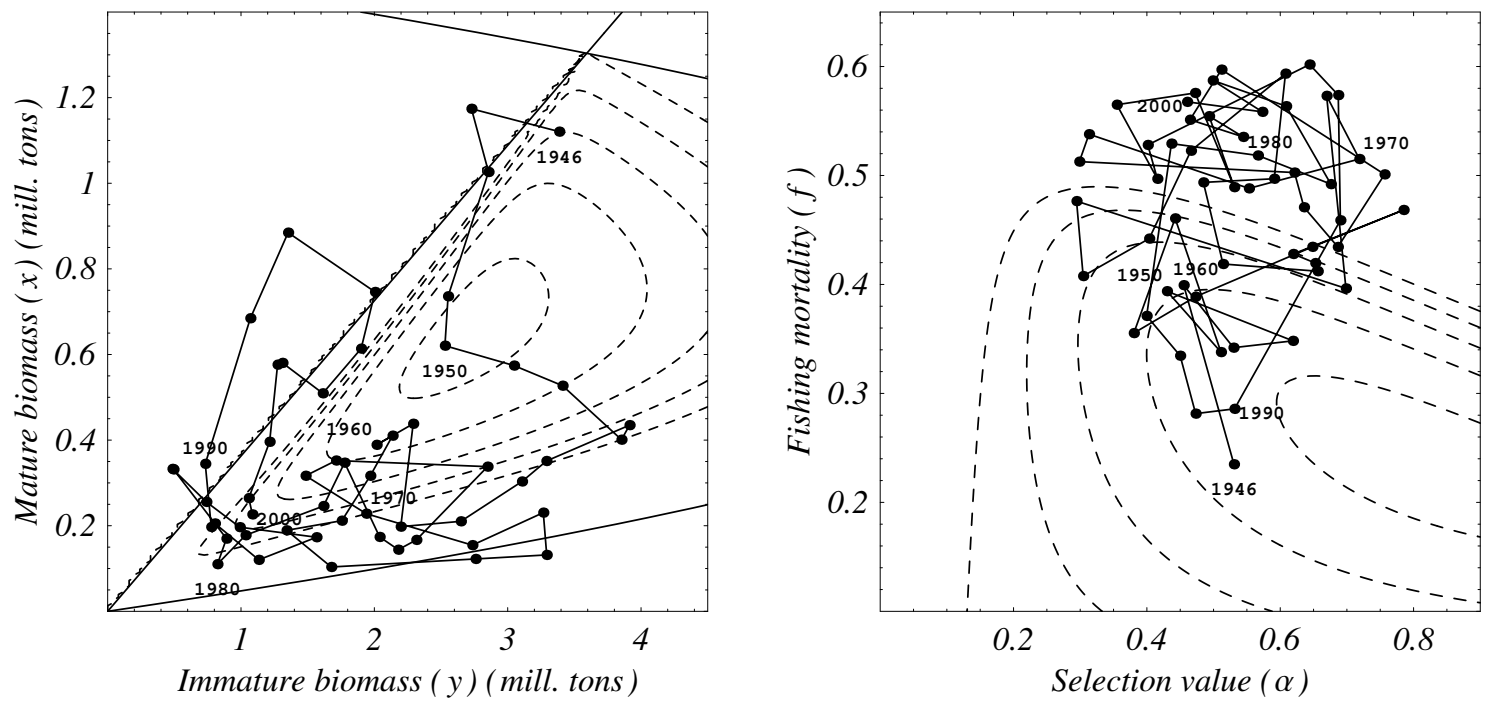

Fig. (5). Historical data 1946-2000 from Table 1 (solid lines) placed in the phase plot of the system from Fig. (2) and (4) (left) and the contour plot from Fig. (3) (right). The dashed isocurves indicate resource rent equilibriums of $0,1,2,3$ and 4 billion NOK.

Table 5. Sensitivity analysis on the impact choice of parameter value of $c_{2}$ has on the two control variables $\alpha$ and $f$ and the state variables $x$ and $y$. All values in percentage change from three of the basic runs in Table 4, namely the $\delta$ values $0,0.05$ and infinity.

\begin{tabular}{|c|c|c|c|c|c|c|c|}
\hline \multirow{2}{*}{$\delta$} & \multirow{2}{*}{ 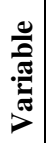 } & \multicolumn{6}{|c|}{ Percentage change in $c_{2}$ value } \\
\hline & & -50 & -10 & -1 & +1 & +10 & $+\mathbf{5 0}$ \\
\hline \multirow{5}{*}{0} & $\alpha$ & 16.2 & 1.9 & 0.2 & -0.2 & -1.5 & -5.5 \\
\hline & $f$ & $-14 \cdot 3$ & -1.5 & -0.1 & 0.1 & 1.2 & 4.2 \\
\hline & $x$ & -0.1 & -0.0 & -0.0 & 0.0 & 0.0 & 0.0 \\
\hline & $y$ & -7.9 & -0.8 & -0.1 & 0.1 & 0.6 & 2.1 \\
\hline & $\pi$ & -1.7 & -0.2 & -0.0 & 0.0 & 0.1 & 0.5 \\
\hline \multirow{5}{*}{.05} & $\alpha$ & 6.5 & 0.8 & 0.1 & -0.1 & -0.6 & -2.3 \\
\hline & $f$ & -5.4 & -0.6 & -0.1 & 0.1 & 0.5 & 1.8 \\
\hline & $x$ & -0.1 & -0.0 & -0.0 & 0.0 & 0.0 & 0.0 \\
\hline & $y$ & -2.3 & -0.3 & -0.0 & 0.0 & 0.3 & 0.9 \\
\hline & $\pi$ & -1.1 & -0.1 & -0.0 & 0.0 & 0.1 & 0.4 \\
\hline \multirow{5}{*}{$\infty$} & $\alpha$ & $-13 \cdot 2$ & -1.5 & -0.2 & 0.1 & 1.2 & 4.8 \\
\hline & $f$ & 9.5 & 1.2 & 0.1 & -0.1 & $-1-0$ & -3.9 \\
\hline & $x$ & -0.5 & -0.1 & -0.0 & 0.0 & 0.1 & 0.5 \\
\hline & $y$ & 6.8 & 0.9 & 0.1 & -0.1 & -0.7 & -2.8 \\
\hline & $\pi$ & 0.0 & 0.0 & 0.0 & 0.0 & 0.0 & 0.0 \\
\hline
\end{tabular}

Numerical solutions $\alpha^{*}$ and $f^{*}$ of $(22 \mathrm{a}, \mathrm{b})$ for different values of the discount rate $\delta$ are shown in Table 4, where estimated values of $r, a, s, b$ and $k$ from Table 2 and estimated prices and cost parameters $p_{1}, p_{2} \quad c_{1}$ and $c_{2}$ from Table 3 , have been applied. The numerical solutions are also shown together with a contour plot of total profit in Fig. (3), as a curve connecting different $f$ - $\alpha$ combinations of maximum profit for varying values of the discount rate $\delta$.

As pointed out in the previous part, a selection parameter $\alpha=1$ (no selection) is expected in the case of open access, since possible benefits of choosing other values partly will be future benefits, but more important, benefits that have to be shared with others exploiting the fish stock resource. This causes a fundamental difference between the open access equilibrium solution and the optimal equilibrium solution where no value is put on future benefits $(\delta=\infty)$ as seen in Table 4 and in Fig. (3) and (4).

As a high degree of uncertainty is related to the values of the parameters in the cost equation, a sensitivity analysis on the effect the value of $c_{2}$ had on equilibriums found in Table 4 was performed, of which the results are displayed in Table 5.

\section{DISCUSSION}

Historical fluctuations in the Northeast Arctic Cod fishery are reasonably well explained by the varying fishing effort from year to year in a simple growth model including cannibalistic behaviour. The symbiosis or cannibalism model (1), (2) describes coexistence of the immature and mature fractions of a population, both benefiting and depending of each other, at the same time as immature are preyed upon by mature fraction. The five model parameters 
whereby one $(k)$ expresses the cannibalistic relationship are lumped based parameters covering a range of different biological processes. Two parameters $(a$ and $b)$ are closely related to recruitment relationships between the two fractions, maturation and indirectly by that also cannibalism. Also the intrinsic growth rates ( $r$ and $s$ ) are lumped based parameters covering individual growth, density dependency, mortalities, etc.

The economic model summed up by the resource rent equation (15) is similarly simple, including a cost of selective fishery. Selective fishing is allowed to work both ways, selecting out mature or immature fish in catch. While reducing the catch of immature fish, a minimum selection value of no immature catch is reached at $\alpha=0$. The rationale behind enforcing $\alpha$-values larger than 1 is that investment in selective fishery by avoiding catching immature fish, over time increases the available mature part of the stock, as more immature fish survive this period.

The Northeast Arctic Cod fishery is a fishery where it has been put a lot of effort into protecting juveniles and other immature cod from being caught. Consequently the $\alpha$-value is expected to be significantly less than one. It should however be noted that the management objectives may not be the only explanation of the historical record seen in Table 4 , as the relative density of mature fish is higher on the spawning grounds where a large share of the fish is caught [4]. This may question if the cost equations employed here should have its lowest unit cost at a value closer to the historical average rather than at $\alpha=1$.

The results in Table 4 show how the social optimal $\alpha$-value vary with the social discount rate $\delta$. No selection, or rather negative selection ( $\alpha$-values higher than 1), is optimal at discount rates above $10 \%$, while a full selective fishery $(\alpha=0)$ is never found to be optimal at any discount rate. The latter also turns out to be valid in the special case of $c_{2}=0$ (no cost of selection). Equilibriums by other values of $c_{2}$ have been studied in a sensitivity analysis (Table 5), indicating the same threshold value close to a discount rate of $10 \%$ where the optimal selection is no selection. Reduced selection cost below a discount rate of $10 \%$ gives increased selection and reduced fishing effort in optimum equilibrium solution. The selection cost parameter does however not alter the general conclusions as these seem to be rather robust towards such changes.

Open access equilibrium is supposed to imply a non-selective fishery $(\alpha=1)$. The catch history and fishing on spawning grounds indicate however that the $\alpha$-value in open access equilibrium more probably will be found between 0.5 and 1.0 and may even be below the $\alpha$-value of maximum sustainable economic yield, which is 0.86 ( $\delta=0$ in Table 4 ). Open access equilibrium is therefore likely to be found even further from the optimal solution of $\delta=\infty$ (Table 4) than indicated in our results. In that case investments in mesh size regulations on nets and trawls and minimum size regulation on fish caught may be inconsistent with the objective of social optimal regulation.

As seen in Fig. (5b) the selection factor $(\alpha)$ in the period 1946-2000 varied between 0.3 - 0.6 with a mean value of $\alpha=0.5316$ (Table 4). Mesh size regulation was first introduced in the $1960 \mathrm{~s}$, but the low selection factor value at least in the early years reflects the fishing pattern referred to above; the winter-spring fishery on the coastal spawning grounds being the most important. Juveniles of the migrating cod stock are available in the open sea fisheries, mainly exploited by trawlers. Since today's selection factor is far below calculated optimal values of any level of discount rates, it may be argued that relatively the open sea fishery should increase their share in order to obtain optimal exploitation. Less surprising, Table 4 also shows that current fishing mortality rate on mature cod is much higher than what is found to be the social optimal fishing mortality rate (at equilibrium), independent on discount rate value. At a discount rate of 5\%, optimal fishing mortality rate of mature cod is close to the fishing mortality rate of immature cod in year $2000(\alpha f)$, which actually is close to the optimal equilibrium value, as this optimum in practical terms is a non-selective fishery.

How far the actual fishery has been from an equilibrium situation is indicated by the two panels in Fig. (5), both showing equilibrium iso-resource rents as functions of combinations of stock biomasses (Fig. 5a) and combinations of selection and fishing mortality rates (Fig. 5b). In the first case the changes in stock sizes pass through the area of maximum resource rent before settling down at a lower stock size area. Fig. (5b) shows however, that the maximum resource rent in equilibrium is far from the historical values of the selection parameter and fishing mortality rates over the period.

Based on these findings it is reasonable to assume a twofold management problem, aiming to maximise the present value of all future resource rent from the fishery; 1) Reducing the overall fishing mortality, and 2) Changing the selection pattern towards a less selective fishery, relatively increasing the share placed on the immature fraction of the stock. 


\section{REFERENCES}

[1] Myers RA, Blanchard W, Thompson KR. Summary of North Atlantic fish recruitment 1942-1987. Can. Tech. Rep. Fish. \& Aquat. Sci. 1990; 1743.

[2] Hjort J. Fluctuations in the great fisheries of Northern Europe. Const. Int. Explor.-Mer., Copenhagen. 1914.

[3] Øiestad V. Historic changes in cod stocks and cod fisheries: Northeast Arctic cod. ICES mar.Sci. Symp. 1994; 198: 1730.

[4] Anon. Advisory Committee on Fisheries Management (ACFM): Report of the Arctic fisheries Working Group. ICES CM 2001/ACFM:19.

[5] Bogstad B, Lilly GR, Mehl S, Pálsson ÓK, Stefánsson G. Cannibalism and year-class strength in Atlantic cod (Gadus morhua) in Arcto-boreal ecosystems (Barents Sea, Iceland, and eastern Newfoundland). ICES Mar.Sci.Symp. 1994; 198: 576-599.

[6] Wikan A, Eide A. An analysis of a nonlinear stagestructured cannibalism model with application on the Northeast Arctic cod stock. Bull. Math. Biol. 2004; 66: 1685-1704.

[7] Wikan A. From chaos to chaos. An analysis of a discrete age-structured prey-predator model. Journal of Mathematical Biology. 2001; 43(6): 471-500.

[8] Gurtin ME, Levine DS. On populations that cannibalize their young. SIAM J. Appl. Math 1982; 42: 94-108.

[9] Cushing JM. A simple model of cannibalism. Math. Biosc. 1991; 1007: 47-72.

[10] Van den Bosch F, Gabriel W. Cannibalism in an agestructured predator-prey system. Bull. Math. Biol. 1997; 59(3): 551-567.

[11] Magnússon K. Destabilizing effect of cannibalism on a structured predator-prey system. Math. Biosc. 1999; 155: 61-75.

[12] Kohlmeier, Ebenhöh. The stabilizing role of cannibalism in a predator-prey system. Bull. Math. Biol. 1995; 57: 401411

[13] Hannesson R. Optimal harvesting of ecologically interdependent fish species, Journal of Environmental Economics \& Management 1983; 10: 329-345.

[14] Ragozin DL, Brown G. Harvest policies and nonmarket valuation in a predator - prey system, Journal of Environmental Economics \& Management 1985; 12: 155168.

[15] Wilen J, Brown G. "Optimal recovery paths for perturbations of trophic level bioeconomic systems", Journal of Environmental Economics and Management, 1986; $13: 225-234$

[16] Flaaten O. Bioeconomics of sustainable harvest of competing species, Journal of Environmental Economics \& Management 1991; 20: 163-180.

[17] Brock BW, Xepapadeas A. Management of Interacting Species: Regulation under Nonlinearities and Hysteresis", Resource and Energy Economics. 2004.

[18] May R, Beddington JR, Clark CW, Holt SJ, Laws RM. Management of multispecies fisheries. Science 1979; 205: 267-277.

[19] Eide A. Shrimp or cod? A bioeconomic analyses of the cod and shrimp stocks of the Barents Sea. Thesis for the Master degree in Fisheries Science, NFH, Troms $\emptyset$. (In Norwegian). 1987.

[20] Armstrong CW. Sharing a Fish Resource - Bioeconomic Analysis of An Applied Allocation Rule. Environmental and Resource Economics 1999; 13: 75-94.

[21] Armstrong CW, Sumaila UR. Cannibalism and the optimal sharing of the North-East Atlantic Cod stock: A bioeconomic model. J. Bioeconomics 2000; 2: 99-115.

[22] Armstrong CW, Sumaila UR. Optimal allocation of TAC and the implications of implementing an ITQ management system for the North-East Arctic cod. Land Economics 2001; 77: 350-359. 